\title{
3D MODELING WITH PHOTOGRAMMETRY BY UAVS AND MODEL QUALITY VERIFICATION
}

\author{
V. Barrile ${ }^{a}$, G. Bilotta ${ }^{\text {b, }}{ }^{*}$, A. Nunnari ${ }^{\text {a }}$ \\ ${ }^{a}$ Geomatics Lab, DICEAM, Università Mediterranea di Reggio Calabria, 89123 loc. Feo di Vito, Reggio Calabria, Italy - \\ vincenzo.barrile@unirc.it, ninonunnari@hotmail.it \\ ${ }^{\mathrm{b}}$ Dept. of Planning, IUAV University of Venice, Santa Croce 191, Tolentini 30135 Venice, Italy - \\ giuliana.bilotta@iuav.it
}

KEY WORDS: 3D modeling, Structure from motion, Laser scanner, Monitoring, UAV

\begin{abstract}
:
This paper deals with a test lead by Geomatics laboratory (DICEAM, Mediterranea University of Reggio Calabria), concerning the application of UAV photogrammetry for survey, monitoring and checking. The study case relies with the surroundings of the Department of Agriculture Sciences. In the last years, such area was interested by landslides and survey activities carried out to take the phenomenon under control. For this purpose, a set of digital images were acquired through a UAV equipped with a digital camera and GPS. Successively, the processing for the production of a 3D georeferenced model was performed by using the commercial software Agisoft PhotoScan. Similarly, the use of a terrestrial laser scanning technique allowed to product dense cloud and 3D models of the same area. To assess the accuracy of the UAV-derived 3D models, a comparison between image and range-based methods was performed.
\end{abstract}

\section{INTRODUCTION}

The realization of a photogrammetric survey, with the subsequent creation of three-dimensional photo-realistic models, has involved researchers from various branches of the scientific world for years.

The introduction of Unmanned Aerial Vehicle (UAV) systems, coupled with modern processing techniques, allows taking aerial photographs with important advantages such as: the high execution speed of the survey the remarkable detail of the images obtained and the lesser expense, economical and in terme of time, compared to traditional techniques. The ability to set flight plans, appropriately established at the preliminary stage as a function of the study object, and the growing availability of hardware and software systems to improve their automation, make drones a state-of-the-art tool for monitoring and control activities also involving areas of significant extension.

\section{STUDY AREA}

The experimentation was conducted within the university campus of Reggio Calabria, located in Feo di Vito, precisely in the area close to the facades of the former faculties of Engineering and Agrarian Science, Figure 1.

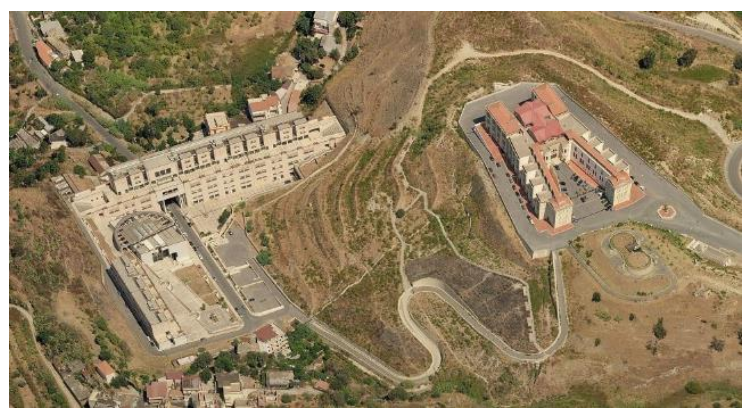

Figure 1. Aerial view of the area

The aim of this work is to monitor and control the deformations of a part of the hill on which the Agrarian Department is located, Figure 2. This structure, after its construction, was affected by cracking and visible cracks, caused by landslides.

For the test, we used two data capture techniques, which are different from each other for capture methodologies, processing times and costs.

\section{SURVEY BY UAV}

The photogrammetric imaging with UAV platform cannot be without planning to capture a block of images with sufficient coverage (longitudinal and transverse) and a ground sample distance (GSD) dictated by the application type. The presence of an autopilot system (with GPS) is undoubtedly an essential requirement for capturing images that reflect the characteristics of a photogrammetric block. The autopilot function is functional to define and follow block cracks and image capture centres. Shots are performed at positions previously established or, commonly, at regular intervals. Flight data are recorded for the next phase of processing and aerial triangulation.

\footnotetext{
* Corresponding author
} 


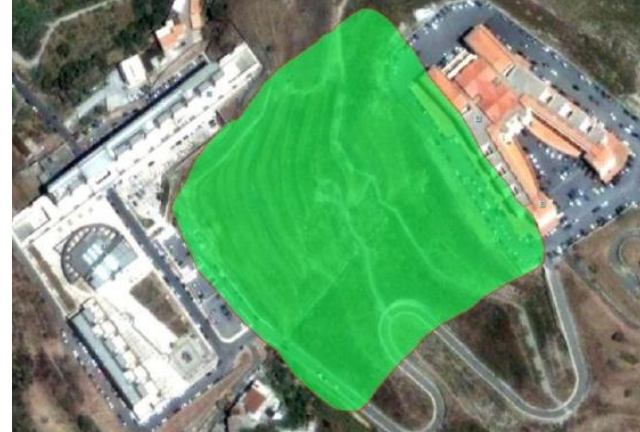

Figure 2. Extension of the study area

Traditionally in the photogrammetric technique, the camera was calibrated or pre-calibrated.

In fact, a self-calibration procedure would require the use of transverse strips, possibly at different dimensions. However, the evolution of software has enabled the use of pre-calibration cameras, since within the software itself there is a database with the characteristics of the most popular cameras in commerce. The images taken during the flight are then processed following the classic photogrammetric procedure.

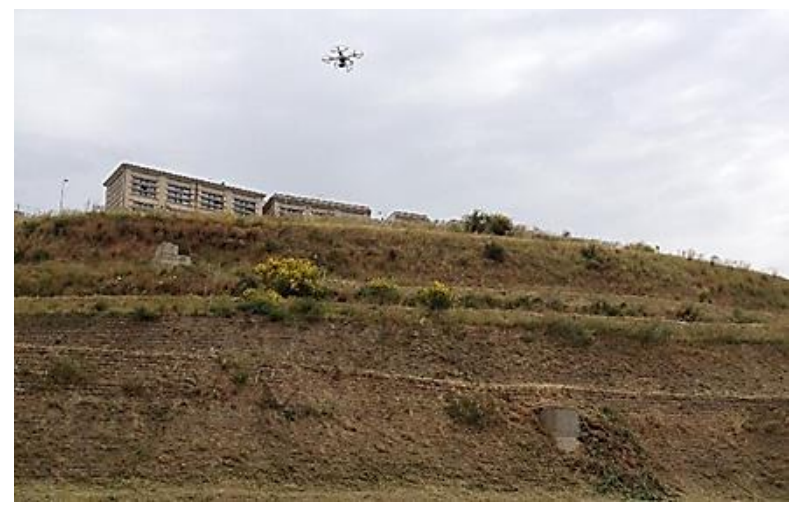

Figure 3. UAV flying in survey phase

Usually, in flights executed through UAV, direct image orientation is not advisable, since the use of GPS, with only the code signal, makes the orientation data totally inadequate for this procedure. The bundle block adjustment is thus done by automatically identifying the homologous points and by defining a relative or absolute reference system (geo-reference), locating on the ground some points clearly visible inside the shots and determining their coordinates with a suitable topographic tool, thus ensuring due precision. Image orientation can be derived from point clouds, derived orthophotos, polygonal, textured 3D models, or subject matter information, and extracting surface imaging (DSM) or soil (DTM) models with image matching techniques.

Monitoring of the area under investigation was carried out using the Dji Phantom 2 Vision Plus drone, a compact sized quadcopter equipped with a GoPro HERO3+ Silver Edition video camera capable of taking 10 Megapixel photos and video shooting possibilities Full HD 1920x1080p at $30 \mathrm{fps}$ and 720p at $60 \mathrm{fps}$, Figures 3 and 4 . The angle of the field of view, depending on the choosed image format, is $110^{\circ}$ or $85^{\circ}$.

The creation of the 3D point cloud obtained from photogrammetric data was made through the PhotoScan software produced by Agisoft LLC, which allows the generation of dense clouds, meshes and textures (Barazzetti, Scaioni, 2010).

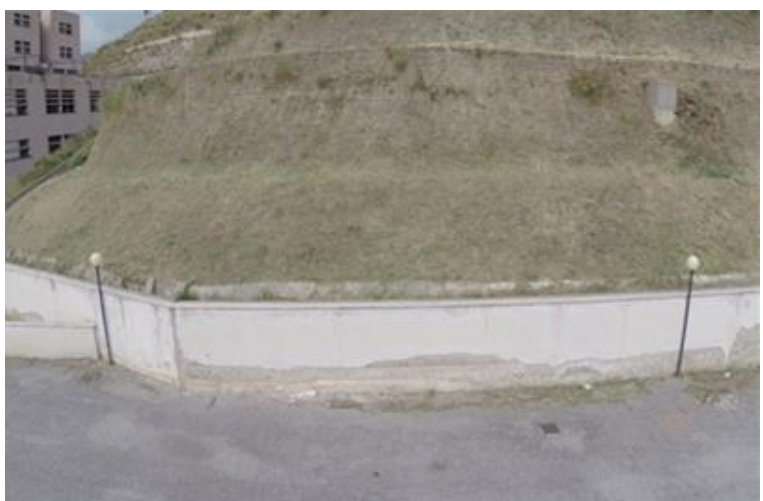

Figure 4. Example of image shot by UAV

Agisoft PhotoScan is a low-cost commercial software that allows to get a high quality 3D model. The workflow is fully automated both for image orientation and for generating and reconstructing the model. The generated model can be exported to be managed and eventually processed with external software. This has led to optimization of processing times, ensuring good performance of the machine / software complex. All processes can be performed with various levels of precision; furthermore, the different parameters can be set in order to improve the final result.

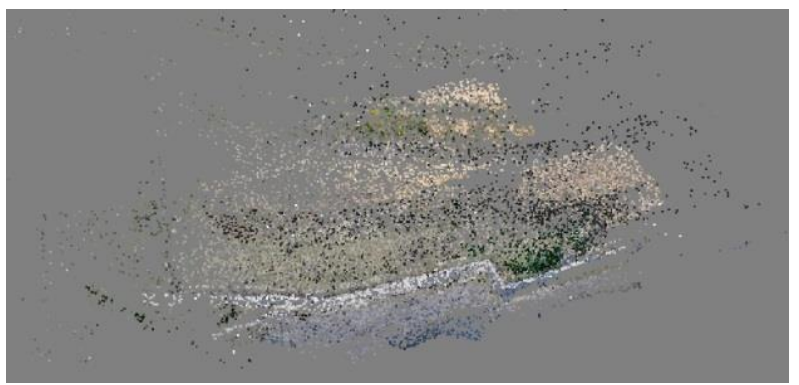

Figure 5. Sparse cloud of the area under examination

The images captured by the GoPro HERO3+ digital camera have been saved in .jpeg format, file format supported by the software used in the test. For processing images, an HP Z800 workstation was used, with two Xeon Hexa Core X5650 CPUs, 64GB RAM memory and two NVIDIA Quadro FX 4800 video cards of $1.5 \mathrm{~GB}$ each.

The processing phases were as follows:

- Align photos (photo alignment) that consists in identifying the tie points through the use of operators of interest. The points chosen in the different pictures must have common features to be adequately superimposed. For good performance, the image quality must be high; there should be few shadow areas and adequate lighting. At this stage, the cloud of scattered points is obtained (Figure 5);

- Build dense clouds. Through this phase, a dense cloud is constructed using dense image matching algorithms (Figures $6 \mathrm{a}$ and $6 \mathrm{~b}$ ). These are subdivided into algorithms that use a stereo pair to find matches (stereo matching) and those that instead identify them in multiple images (stereo multi-view); 

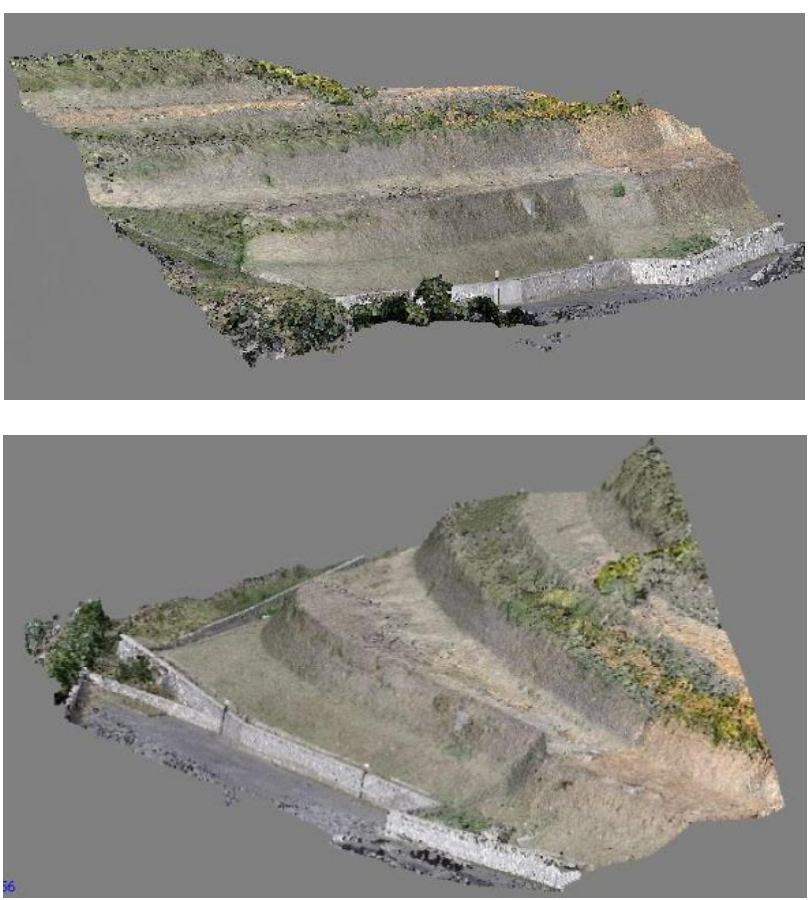

Figures $6 \mathrm{a}$ and $6 \mathrm{~b}$. Different angles of the dense cloud

- Build mesh, which consists of generating a polygonal pattern based on the dense cloud created. The mesh is a subdivision of a solid in solid particles of polyhedra, Figure 7;

- Build texture, it allows instead of getting the 3D representation of the work under investigation, Figure 8.

In total, during the entire flight session for DEM production (Figure 9), were performed 371 photographic shots.

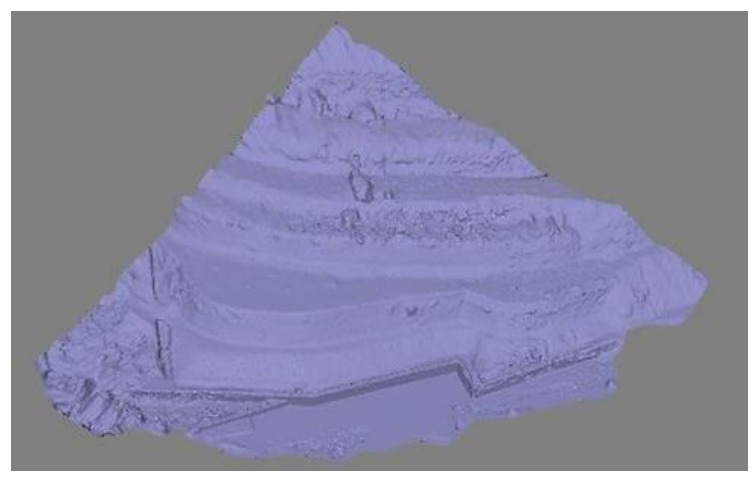

Figure 7. Mesh obtained

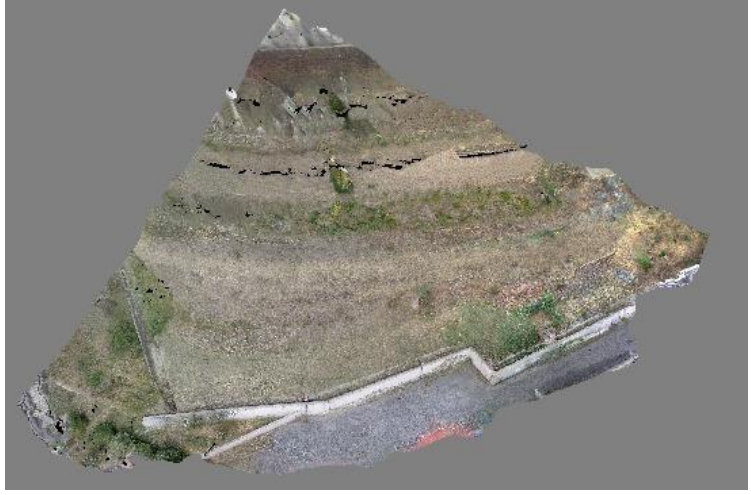

Figure 8. Textured model

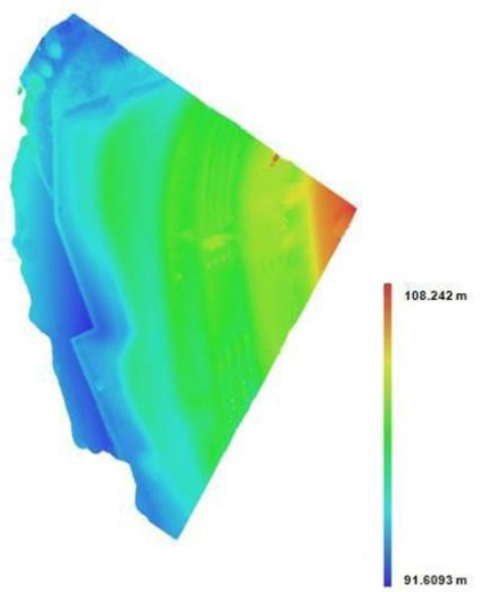

Figure 9. Generation of DEM

The computerized processing procedure from frames to model 3D (at each moment) is carried out according to a predetermined order in different phases (spatial modeling). The first phase is that of alignment of the images and of the construction of the sparse point cloud (cloud points basis). This step identifies the common points in both frames using the image matching algorithms. The points selected in the various photos must necessarily have some features in common for properly overlapping. The result is an overall view of all shooting points positioned along the three Cartesian axes (local reference system) and the points constituting the sparse points cloud. The construction of the denser point cloud (dense cloud points) represents the second stage of processing, in which starting from the cloud of sparse points we get a more detailed and dense cloud of points. The realization of the mesh allows obtaining a polygonal mesh of the sea surface, while the construction of the texture is the last step that allows to "coating" the polygonal mesh generated in the previous step using the color data of the uploaded images. The texture is, in fact, a set of points, lines, and shapes that give the overall impression of a compact surface, making it as realistic 3D model so far obtained. The $3 \mathrm{D}$ model with texture obtained is subsequently scaled and geo-referenced using data collected on the structure supporting the camera site (Barrile, Bilotta, Pozzoli, 2017).

The temporal combination of the obtained models (space-time modeling) is made by appropriate algorithm implemented in own on MATLAB. 


\section{SURVEY BY TLS}

The use of terrestrial laser scanners has the ability to provide useful data for geometric modelling and determination (by comparison of the model realized in successive epochs) of deformations that are created. Laser technology, bypassing cases of simpler realization where the magnitude of deformations to be evaluated is of greater magnitude than measurement uncertainty, is useful and productive in most engineering applications that require measurements near-deformation, if not less than the uncertainty of scanned data.

For scanning the area, we used a Riegl LMS-Z420i terrestrial scanner (Fig. 10) (Barrile, Bilotta, Meduri, 2014), (Barrile, Bilotta, 2014).

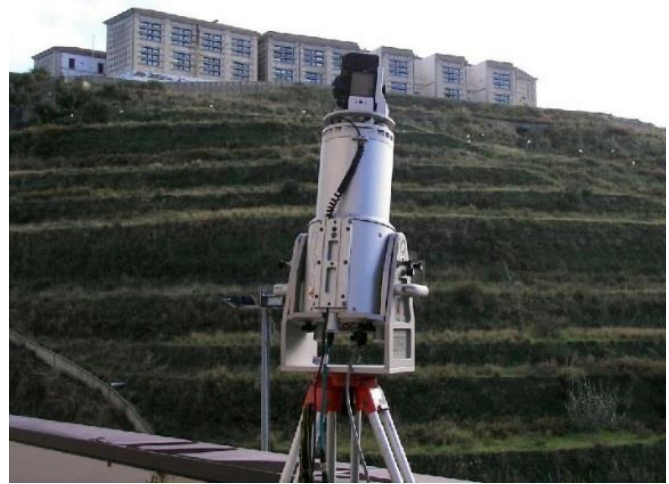

Figure 10. TLS and survey area

The Riegl LMS-Z420i series automatically associates scanning with high-resolution images captured by the metric camera installed and calibrated, allowing to leverage high definition images for macro fracture detection and digitization and great detail details. The laser scanner's external metric camera allows easy upgrading of the photographic part (sensor resolution) and the choice of the best optic depending on the type of work. The ability to mount tele-lens lenses allows for high-definition texture to detect morphologies (Barrile et al., 2016).

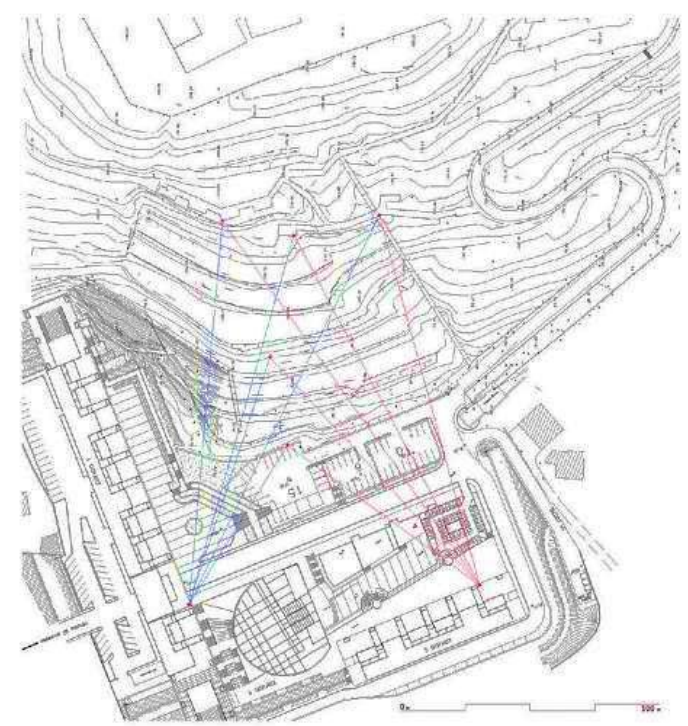

Figure 11. Plane area concerned with indication of station points

The survey of the hill in question (Barrile, Bilotta, Meduri, 2009) is structured with the choice of instrument positions, its set-up, and its calibration (see Figures 11 and 12). Data Acquisition and Data Acquisition is done by recording point clouds, filtering, data cleansing, modelling and creating three-dimensional mesh (Barrile, Bilotta, Meduri, 2014), Figures 13, 14 and 15. The study area occupies $23,900 \mathrm{~m}^{2}$.

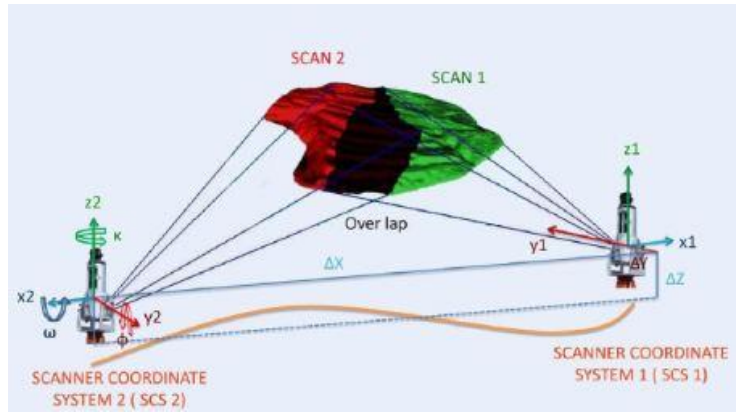

Figure 12. Survey Scheme

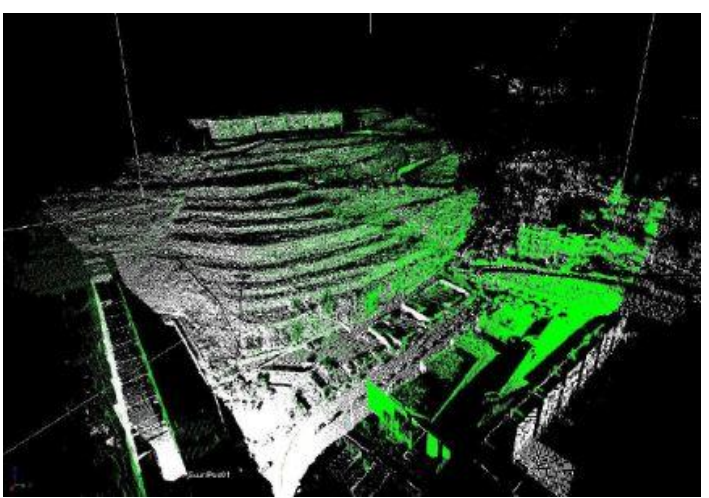

Figure 13. Dense point cloud obtained

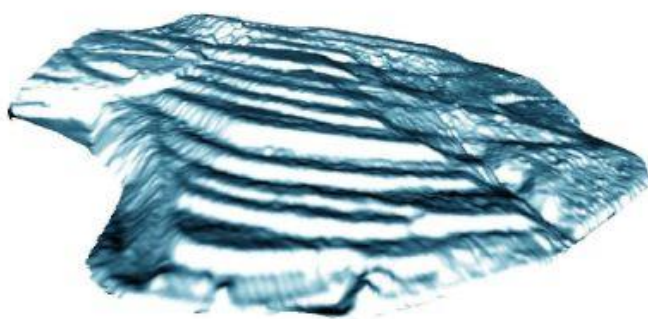

Figure 14. Mesh obtained

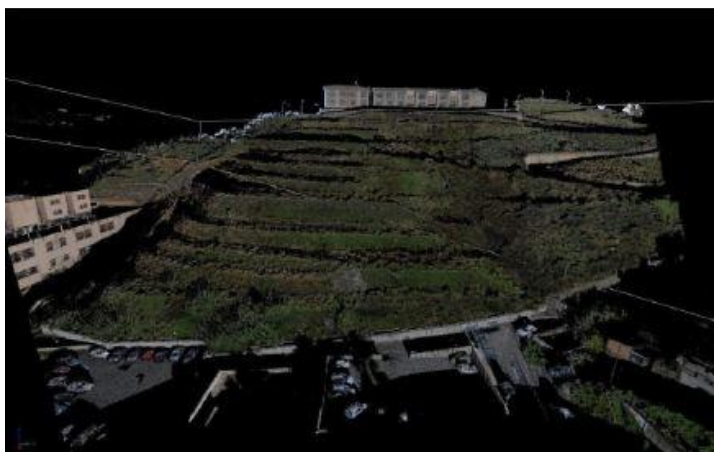

Figure 15. Textured model 


\section{COMPARISON BETWEEN THE TWO TECHNIQUES}

The comparison between these two techniques was performed by identifying and detecting, by GPS, ten "significant" points, thus obtaining the coordinates and their altitudes. After completing the laser scanning and drone/computer vision measurements and creating their respective DEMs, the dimensions of both $3 \mathrm{D}$ models related to the "significant" points detected by GPS methodology were analysed, Figure 16. The topographic survey is performed with the Leica Viva GNSS GS15 rover in RTK mode.

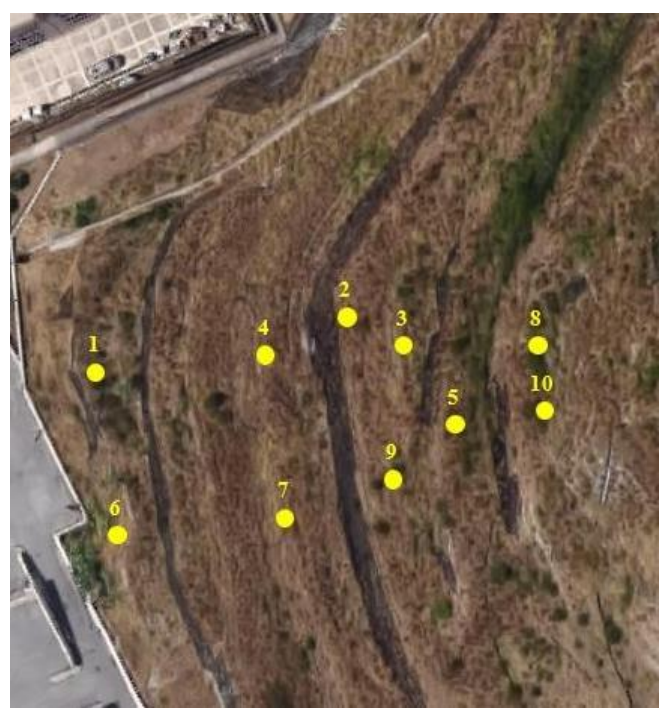

Figure 16. Points detected by GPS

The following tables (1 and 2) and Figure 17 summarize the main results obtained by using the two different survey methodologies.

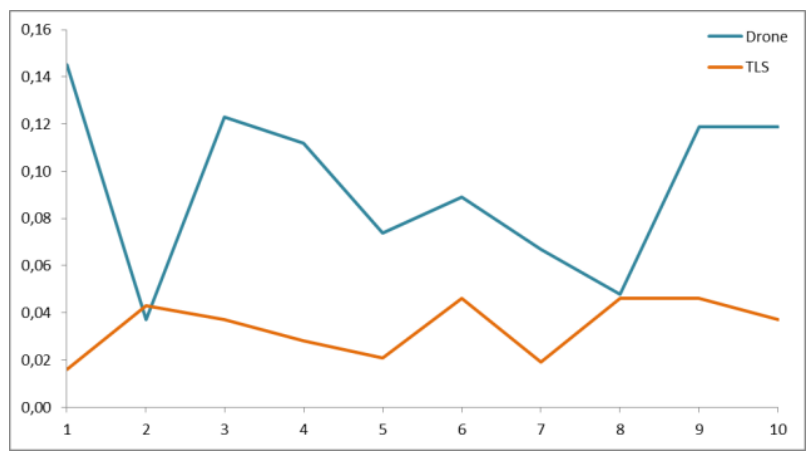

Figure 17. Comparison of the errors $(\mathrm{cm})$ of the two systems

\begin{tabular}{|l|r|}
\hline $\mathrm{N}^{\circ}$ Shots & 371 \\
\hline Acquisition Time & $0,25 \mathrm{~h}$ \\
\hline Processing Time & $5,75 \mathrm{~h}$ \\
\hline Dense Cloud & 105841202 \\
\hline No. Vertices & 5614879 \\
\hline No. Faces & 2807440 \\
\hline Average of Errors (in absolute value) & $9 \mathrm{~cm}$ \\
\hline Standard Deviation $\sigma$ & $12 \mathrm{~cm}$ \\
\hline
\end{tabular}

Table 1 . Summary of results obtained by drone

\begin{tabular}{|l|r|}
\hline Processing Time & $3,80 \mathrm{~h}$ \\
\hline Dense Cloud & 91876298 \\
\hline No. Vertices & 4986234 \\
\hline No. Faces & 2265345 \\
\hline Average of Errors (in absolute value) & $3,5 \mathrm{~cm}$ \\
\hline Standard Deviation $\sigma$ & $5 \mathrm{~cm}$ \\
\hline
\end{tabular}

Table 2. Summary of results obtained by TLS

\section{CONCLUSIONS}

The present experiment showed that, besides the Laser Scanner Systems, even the lowest cost systems based on UAV image processing and photogrammetric analysis with Structure from Motion algorithms are able to produce 3D models of areas (also large areas), with a good level of accuracy. Data acquisition with remote pilot systems is faster, more flexible, and cost-effective than laser scanning.

The results achieved with the PhotoScan software show centimetric standard deviations, highlighting how, from a geometric point of view, they are very close to the data obtained by laser scanning.

Moreover, it is good to note that weather conditions, in particular the presence of a strong wind, have not allowed the UAV to reach such a height that it can obtain the best data possible, limiting in fact the results achieved.

Quality and reliability are influenced, in particular, and especially for large items, by light conditions, number of images, and, of course, frame resolution. Measurement techniques and identification of control points are of great importance in order to scale the obtained 3D model. The use of a high-resolution camera with optics suitable for detection purposes could improve the results achieved in this work. Of great importance are the performance capabilities of computers, especially for larger objects, characterized by a large number of shots essential for proper reconstruction of the object acquired.

\section{REFERENCES}

Barazzetti, L., Scaioni, M., 2010. Dalle immagini dell'oggetto alla replica fisica in modalità automatica. In: Atti 14 Conferenza Nazionale ASITA, Brescia, 9-12 november 2010, pp. 149-154.

Barrile, V., Meduri, G. M., Bilotta, G., 2009. Laser scanner surveying techniques aiming to the study and the spreading of recent architectural structures. In: Recent Advances in Computer Engineering, Proceedings of the 9th WSEAS International Conference on Signal, Speech and Image Processing, SSIP '09, Budapest. pp. 92-95.

Barrile, V., Meduri, G. M., Bilotta, G., 2011. Laser scanner technology for complex surveying structures. Wseas transactions on signal processing, Vol. 7, pp. 65-74.

Barrile, V., Meduri, G.M., Bilotta, G., 2013. Least squares 3D algorithm for the study of deformations with Terrestrial Laser Scanner, Recent Advances in Electronics, Signal Processing and Communication Systems, Proceedings EUROPMENT 2013 International Conference on Electronics, Signal Processing and Communication Systems, ESPCO 2013, Venice, Italy, Sept. 2830, 2013, pp. 162-165.

Barrile, V., Meduri, G.M., Bilotta, G., 2014. An application of the Least Squares 3D algorithm for territorial monitoring and

\begin{tabular}{|l|r|}
\hline $\mathrm{N}^{\circ}$ Shots & 2 \\
\hline Acquisition Time & $1,45 \mathrm{~h}$ \\
\hline
\end{tabular}


control, International Journal of Systems Applications, Engineering \& Development, Vol. 8, pp. 18-25.

Barrile, V., Meduri, G.M., Bilotta, G., 2014. Comparison between two methods for monitoring deformation with Laser Scanner, WSEAS transactions on signal processing, Vol. 10.

Barrile, V., Bilotta, G., Meduri, G.M., 2014. The TLS to study deformations using ICP algorithm. Recent Advances in Electrical and Computer Engineering - Proceedings of the 2014 International Conference on Circuits, Systems, Signal Processing, Communications and Computers (CSSCC 2014), Venice 15/03/2014 - 17/03/2014, p. 40-44.

Barrile, V., Bilotta, G., 2014. Self-localization by Laser Scanner and GPS in automated surveys. Computational Problems in Engineering. Lecture Notes in Electrical Engineering, Springer, Vol. 307, pp. 293-313.

Barrile, V., Meduri, G.M., Bilotta, G., 2014. Experimentations and Integrated Applications Laser Scanner/GPS for Automated Surveys. Wseas transactions on signal processing, Vol. 10, pp. 471-480.

Barrile, V., Bilotta, G., Lamari, D., Meduri, G.M., 2015. Comparison between techniques for generating 3D models of cultural heritage. In: Proceedings of the 2015 International Conference on Civil Engineering (CIVILENG 2015), Zakynthos Island, Greece, July 16-20 2015.

Barrile, V., Bilotta, G., D’Amore, E., Meduri, G.M., Trovato, S., 2016. Structural Modeling of a Historic Castle Using Close Range Photogrammetry. International Journal of Mathematics and Computers in Simulation, Vol. 10, pp. 370-380.

Barrile, V., Bilotta, G., Meduri, G. M., 2016. Identifying damage on cars through the integrated use of TLS/SfM with thermographic images. International Journal of Circuits, Systems and Signal Processing, Vol. 10, pp. 433-439.

Barrile, V., Bilotta, G., Pozzoli, A., 2017. Comparison between innovative techniques of photogrammetry. In: The 2016 International Conference Applied Mathematics, Computational Science and Systems Engineering, Roma (Italia), 5-7 november 2016, ITM Web of Conferences, Vol. 9, pp. 1-7.

Fourcade, H.G., 1926. A new method of aerial surveying. Trans. of the Royal Society of South Africa 14(1), pp. 93-112.

Hattori, S., Myint, Y., 1995. Automatic Estimation of Initial Approximations of Parameters for Bundle Adjustment. $P E \& R S$, 61(7), pp. 909-915

Longuet-Higgins, H.C., 1981. A computer algorithm for reconstructing a scene from two projections. Nature, 293(10), pp. 133-135.

Pan, H.P., 1999. A Direct Closed-Form Solution to General Relative Orientation of Two Stereo Views. Digital Signal Processing, 9(3), pp. 195-211.

Schut, G.H., 1955. Analytical aerial triangulation and comparison between it and instrumental aerial triangulation. Photogrammetria, Vol. 12, pp. 311-318.

Thompson, E.H., 1959. A rational algebraic formulation of the problem of the relative orientation. Photogrammetric Record, 3(14), pp. 152-159.
Torr, P.H.S., Murray, D.W., 1997. The Development and Comparison of Robust Methods for Estimating the Fundamental Matrix. Int. Journal of Computer Vision, 24(3), pp. 271-300. 